\title{
Outlier-Robust State Estimation for Humanoid Robots*
}

\author{
Stylianos Piperakis ${ }^{1}$, Dimitrios Kanoulas ${ }^{2}$, Nikos G. Tsagarakis ${ }^{2}$, and Panos Trahanias $^{1}$
}

\begin{abstract}
Contemporary humanoids are equipped with visual and LiDAR sensors that are effectively utilized for Visual Odometry (VO) and LiDAR Odometry (LO). Unfortunately, such measurements commonly suffer from outliers in a dynamic environment, since frequently it is assumed that only the robot is in motion and the world is static. To this end, robust state estimation schemes are mandatory in order for humanoids to symbiotically co-exist with humans in their daily dynamic environments. In this article, the robust Gaussian Error-State Kalman Filter for humanoid robot locomotion is presented. The introduced method automatically detects and rejects outliers without relying on any prior knowledge on measurement distributions or finely tuned thresholds. Subsequently, the proposed method is quantitatively and qualitatively assessed in realistic conditions with the full-size humanoid robot WALK-MAN v2.0 and the mini-size humanoid robot NAO to demonstrate its accuracy and robustness when outlier VO/LO measurements are present. Finally, in order to reinforce further research endeavours, our implementation is released as an open-source ROS/C++ package.
\end{abstract}

\section{INTRODUCTION}

Humanoid robot walking constitutes a challenging task and remains up-to-date an open-research problem. Contemporary approaches in motion planning [1], [2], [3] and gait control [4], [5], [6], implicitly or explicitly assume that the humanoid's base information is available in advance. To this end, accurate and robust base state estimation realizes a vital role in dynamic humanoid robot locomotion [7]. Towards that direction, Kuindersma et al. [8], presented a base estimator relying on Newton-Euler dynamics of a floating mass, that effectively combined Inertial Measurement Unit (IMU), kinematics, and LiDAR measurements to estimate the base position, orientation, and velocity. The latter utilized the Error-State Kalman Filter (ESKF), a variant of the Extended Kalman Filter (EKF), carefully crafted to handle the overparametrization of the base orientation. Subsequently, this framework was augmented in [9], [10] to directly account for the visually obtained landscape. In such a way, an ATLAS robot was able to continuously climb up and down staircases. Furthermore, in [11], the same scheme was evaluated with the WALK-MAN robot in a disaster response environment.

Newton-Euler floating mass estimators proved to be effective also in the case of quadruped robots [12]. Bloesch

*This work has been partially supported by the following funded projects: Greek National grand MIS 5030440 vipGPU and the CogIMon (no 644727) EU project.

${ }^{1}$ Stylianos Piperakis and Panos Trahanias are with the Institute of Computer Science, Foundation for Research and Technology - Hellas (FORTH), Heraklion, Greece. \{spiperakis, trahania\}@ics.forth.gr

${ }^{2}$ Dimitrios Kanoulas and Nikos G. Tsagarakis are with the $\mathrm{Hu}-$ manoids and Human-Centered Mechatronics Department, Istituto Italiano di Tecnologia (IIT), Via Morego 30, 16163, Genova, Italy. \{Dimitrios.Kanoulas, Nikos.Tsagarakis\}eit.it.

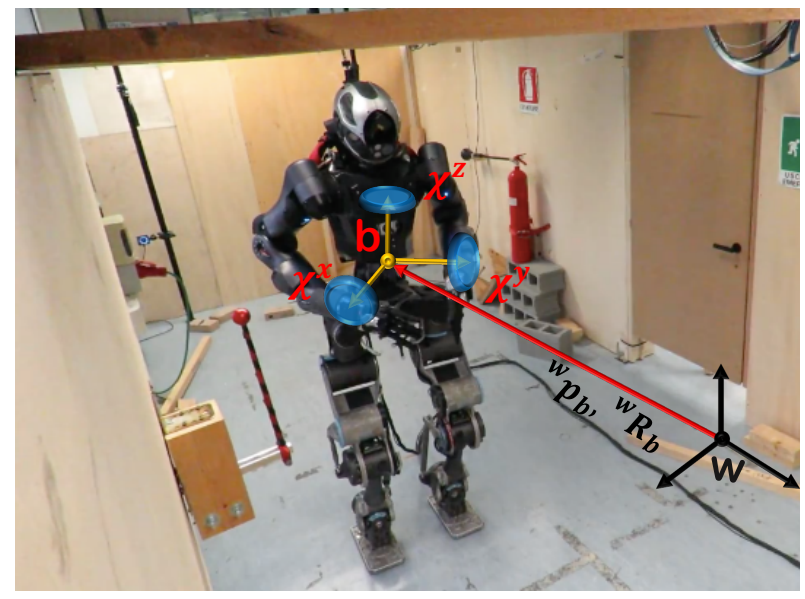

Fig. 1. Illustration of frames used in base estimation on the 29DoF WALKMAN v2.0 humanoid robot: $w$ corresponds to the inertial world frame, $b$ is the base frame, and blue ellipses indicate the orientation uncertainty.

et al. [13], [14], presented a similar approach where the IMU and the kinematically computed feet point contacts were utilized for accurate base estimation with the starlETH quadruped. Rotella et al. [15] appropriately modified the previous scheme for humanoid walking while also considering the feet orientation in the process. Interestingly, it was demonstrated that the base estimation accuracy increases.

Additionally, state-of-the-art 3D-Center of Mass (CoM) estimators [16], [17], [18], derived from consistent dynamics, readily assume that measurements are transformed to an inertial frame of reference before fused. The latter is also common practice in 2D-CoM estimation based on simplified dynamical models [19], [20]. Nevertheless, this dictates that accurate estimates of the base position and orientation are available, otherwise base drift will inevitably propagate to the CoM estimation [21].

\section{A. Problem Statement}

Modern humanoids are commonly employed with cameras and LiDAR sensors to reinforce their perception in unstructured environments. Based on consecutive camera frames one can derive the camera's egomotion with respect to the environment and directly relate it to the robot's motion. In literature this is known as Visual Odometry (VO). Prominent approaches rely on sparse [22] or semi-dense [23] schemes to facilitate real-time execution. Similarly, based on sequential LiDAR scans it is straightforward to match the beams and compute the LiDAR Odometry (LO) [24], [25]. Both approaches are advantageous in the sense that they are unaffected by slippage in uneven/rough terrain when contrasted 
to the kinematically computed leg odometry. However, in all aforementioned schemes, the world is assumed to be mostly static and only the robot is in motion, e.g. the static world assumption. Presumably, this is not the case in human daily environments, due to humans moving along with the robots and/or changing the scene. Hence, the static world assumption is frequently violated. To this end, in order for humanoids to co-exist with humans in a dynamically changing environment it is mandatory to robustify their odometry estimates. Interestingly, in [14] a base estimator with outlier detection for quadruped locomotion was presented. The authors of [14] utilized a probabilistic threshold to quantify weather a measurement is an outlier or not before fusion. Nevertheless, this raises two important questions: a) how can this threshold be determined in advance and b) does this threshold depend on the conditions at hand? Other works [26], not in the scope of base estimation, assumed that the measurements follow a Student-t distribution. Again the obvious question arises whether this is a valid assumption in the case of $\mathrm{VO} / \mathrm{LO}$ measurements.

\section{B. Contribution}

In this work, we propose a novel formulation of the Error-State Kalman Filter (ESKF) which is robust to outlier $\mathrm{VO} / \mathrm{LO}$ measurements that can commonly occur in humanoid walking in dynamic human environments. The contribution to the state-of-the-art is as follows:

- The Robust Gaussian ESKF (RGESKF) is mathematically established based on [8], [27]. More specifically, we present an analytical solution for the general nonlinear Gaussian formulation for outlier detection of [27]. The latter results in a computationally efficient implementation that accomplishes real-time execution.

- The above method does not rely on prior assumptions regarding the measurements probability distributions [26] neither thresholding [14] for the imminent outlier detection.

- We quantitatively and qualitatively assess the proposed method and demonstrate its accuracy and robustness in real-world conditions with two robots, the full-size humanoid WALK-MAN v2.0 [28], and a mini-size NAO humanoid.

- Since this framework relies on sensing that is commonly available on contemporary humanoids and furthermore, is based on generic nonlinear dynamics, we release an open-source ROS/C++ implementation [29] to reinforce further research endeavours.

This article is structured as follows: in Sec. II the proposed Robust Gaussian ESKF is introduced and mathematically established. Subsequently, the proposed scheme is quantitatively and qualitatively assessed in Sec. III and Sec. IV. Finally, Sec. V concludes the article and discusses possible future directions.

\section{BASE Estimation}

Kuindersma et al. [8], presented a base estimator with Newton-Euler dynamics of a floating mass that is effectively used in humanoid walking. At time $t$, the state to be estimated is:

$$
\boldsymbol{x}_{t}=\left[\begin{array}{lllll}
{ }^{b} \boldsymbol{v}_{b} & { }^{w} \boldsymbol{R}_{b} & { }^{w} \boldsymbol{p}_{b} & \boldsymbol{b}_{\boldsymbol{\omega}} & \boldsymbol{b}_{\boldsymbol{a}}
\end{array}\right]^{\top}
$$

where ${ }^{w} \boldsymbol{p}_{b},{ }^{w} \boldsymbol{R}_{b}$ denote the base position and rotation with respect to the world frame $w,{ }^{b} \boldsymbol{v}_{b}$ is the linear velocity, and $\boldsymbol{b}_{\boldsymbol{\omega}}, \boldsymbol{b}_{\boldsymbol{\alpha}}$ are the gyro and accelerometer biases, in the base frame $b$. However, ${ }^{w} \boldsymbol{R}_{b}$ is an overparametrization of the base's orientation. To this end, to track the orientation uncertainty we consider perturbation rotations in the base frame. Thus, if the true base rotation matrix is ${ }^{w} \boldsymbol{R}_{b}$ then ${ }^{w} \boldsymbol{R}_{b}={ }^{w} \hat{\boldsymbol{R}}_{b} e^{\chi}$ where ${ }^{w} \hat{\boldsymbol{R}}_{b}$, is the estimated rotation matrix and $\chi$ denotes the perturbation exponential coordinates. For clarity all aforementioned quantities are depicted in Figure 1.

\section{A. Process Model}

In order to properly define the nonlinear dynamics $\boldsymbol{f}\left(\boldsymbol{x}_{\boldsymbol{t}}, \boldsymbol{u}_{\boldsymbol{t}}, \boldsymbol{w}_{\boldsymbol{t}}\right)$, let ${ }^{b} \overline{\boldsymbol{\omega}}_{b}={ }^{b} \boldsymbol{\omega}_{b}^{\mathrm{imu}}-\boldsymbol{b}_{\boldsymbol{\omega}}$ and ${ }^{b} \overline{\boldsymbol{\alpha}}_{b}={ }^{b} \boldsymbol{\alpha}_{b}^{\mathrm{imu}}-\boldsymbol{b}_{\boldsymbol{\alpha}}$, be the IMU bias-compensated gyro rate and linear acceleration, respectively, then:

$$
\dot{\boldsymbol{x}}_{t}=\overbrace{\left[\begin{array}{c}
\left.-\left({ }^{b} \overline{\boldsymbol{\omega}}_{b}-\boldsymbol{w}_{\boldsymbol{\omega}}\right) \times{ }^{b} \boldsymbol{v}_{b}+{ }^{w} \boldsymbol{R}_{b}^{\top} \boldsymbol{g}+{ }^{b} \overline{\boldsymbol{\alpha}}_{b}-\boldsymbol{w}_{\boldsymbol{a}}\right] \\
{ }^{w} \boldsymbol{R}_{b}\left({ }^{b} \overline{\boldsymbol{\omega}}_{b}-\boldsymbol{w}_{\boldsymbol{\omega}}\right)[\times] \\
{ }^{w} \boldsymbol{R}_{b}{ }^{b} \boldsymbol{v}_{b} \\
\boldsymbol{w}_{\boldsymbol{b}_{\boldsymbol{\omega}}} \\
\boldsymbol{w}_{\boldsymbol{b}_{\boldsymbol{\alpha}}}
\end{array}\right]}^{\boldsymbol{f}\left(\boldsymbol{x}_{\boldsymbol{t}}, \boldsymbol{u}_{\boldsymbol{t}}, \boldsymbol{w}_{\boldsymbol{t}}\right)}
$$

where $\boldsymbol{u}_{t}=\left[\begin{array}{cc}{ }^{b} \boldsymbol{\omega}_{b}^{\mathrm{imu}} & { }^{b} \boldsymbol{\alpha}_{b}^{\mathrm{imu}}\end{array}\right]$ is the input vector, $\boldsymbol{g}$ is the gravity vector, $\boldsymbol{w}_{t}=\left[\begin{array}{llll}\boldsymbol{w}_{\boldsymbol{\omega}} & \boldsymbol{w}_{\boldsymbol{a}} & \boldsymbol{w}_{\boldsymbol{b}_{\boldsymbol{\omega}}} & \boldsymbol{w}_{\boldsymbol{b}_{\boldsymbol{\alpha}}}\end{array}\right] \sim \mathcal{N}\left(\mathbf{0}, \boldsymbol{Q}_{t}\right)$ is the input-process noise that follows a normal zero mean distribution with covariance $Q_{t}$, and [×] is the wedge operation.

Subsequently, denoting the error state vector as:

$$
\boldsymbol{\delta} \boldsymbol{x}_{t}=\left[\begin{array}{lllll}
{ }^{b} \boldsymbol{\delta} \boldsymbol{v}_{b} & \chi & { }^{w} \boldsymbol{\delta} \boldsymbol{p}_{b} & \boldsymbol{\delta} \boldsymbol{b}_{\boldsymbol{\omega}} & \delta \boldsymbol{b}_{\boldsymbol{a}}
\end{array}\right]^{\top}
$$

the error-state dynamics assume the following linear form:

$$
\boldsymbol{\delta} \dot{\boldsymbol{x}}_{t}=\boldsymbol{F}_{t} \boldsymbol{\delta} \boldsymbol{x}_{t}+\boldsymbol{L}_{t} \boldsymbol{w}_{t}
$$

with

$$
\begin{gathered}
\boldsymbol{F}_{t}=\left[\begin{array}{ccccc}
-{ }^{b} \overline{\boldsymbol{\omega}}_{b[\times]} & \left({ }^{w} \boldsymbol{R}_{b}^{\top} \boldsymbol{g}\right)_{[\times]} & \mathbf{0} & -{ }^{b} \boldsymbol{v}_{b[x]} & -\boldsymbol{I} \\
\mathbf{0} & -{ }^{b} \overline{\boldsymbol{\omega}}_{b[\times]} & \mathbf{0} & -\boldsymbol{I} & \mathbf{0} \\
{ }^{w} \boldsymbol{R}_{b} & -{ }^{w} \boldsymbol{R}_{b}{ }^{b} \boldsymbol{v}_{b[x]} & \mathbf{0} & \mathbf{0} & \mathbf{0} \\
\mathbf{0} & \mathbf{0} & \mathbf{0} & \mathbf{0} & \mathbf{0} \\
\mathbf{0} & \mathbf{0} & \mathbf{0} & \mathbf{0} & \mathbf{0}
\end{array}\right] \\
\boldsymbol{L}_{t}=\left[\begin{array}{cc}
-{ }^{b} \boldsymbol{v}_{b[x]} & -\boldsymbol{I} \\
-\boldsymbol{I} & \mathbf{0} \\
\mathbf{0} & \mathbf{0} \\
\boldsymbol{I} & \mathbf{0} \\
\mathbf{0} & \boldsymbol{I}
\end{array}\right]
\end{gathered}
$$

To this end, the ESKF predict step is readily realized as:

$$
\begin{aligned}
\hat{\boldsymbol{x}}_{k}^{-} & =\boldsymbol{f}_{k}^{d}\left(\hat{\boldsymbol{x}}_{k-1}^{+}, \boldsymbol{u}_{k}, \mathbf{0}\right) \\
\boldsymbol{P}_{k}^{-} & =\boldsymbol{F}_{k}^{d} \boldsymbol{P}_{k-1}^{+} \boldsymbol{F}_{k}^{d \top}+\boldsymbol{L}_{k}^{d} \boldsymbol{Q}_{k}^{d} \boldsymbol{L}_{k}^{d \top}
\end{aligned}
$$


where the superscript $d$ indicates the discretized variables at the discrete-time $k$, which are obtained by means of the Euler method for simplicity. Moreover, $\hat{\boldsymbol{x}}_{k}^{-}, \boldsymbol{P}_{k}^{-}$denote the ESKF mean estimate and error covariance respectively, prior to update, while $\hat{\boldsymbol{x}}_{k-1}^{+}, \boldsymbol{P}_{k-1}^{+}$are the same quantities after the update at discrete-time $k-1$.

\section{B. Measurement Model}

The output model of [8], was formulated with the base velocity using the robot's kinematics and the base position, orientation obtained with a Gaussian particle filter on LiDAR measurements, all expressed in the world frame. In this work, besides the kinematically computed base velocity, we consider external measurements of the base position and orientation from either LiDAR Odometry (LO) or Visual Odometry (VO). Nevertheless, such measurements can potentially suffer from outliers in human daily environments due to the static-world assumption, as presented in Sec. IA. Thus, we distinguish the latter with the superscript $o$ for possible outliers as:

$$
\boldsymbol{y}_{k}^{o}=\overbrace{\left[\begin{array}{l}
{ }^{w} \boldsymbol{p}_{b}+\boldsymbol{n}_{\boldsymbol{p}_{b}} \\
{ }^{w} \boldsymbol{R}_{b} \boldsymbol{e}^{\boldsymbol{n}_{b}[\times]}
\end{array}\right]}^{\boldsymbol{h}^{\boldsymbol{o}}\left(\boldsymbol{x}_{\boldsymbol{k}}\right) \boxplus \boldsymbol{n}_{\boldsymbol{k}}}
$$

where $\boldsymbol{n}_{k}=\left[\begin{array}{ll}\boldsymbol{n}_{\boldsymbol{p}_{b}} & \boldsymbol{n}_{b}\end{array}\right]$ denote the external position and orientation measurement noise that follows normal zero mean distribution with covariance $\boldsymbol{R}_{k}^{o}$. The operator $\boxplus$ denotes the proper summation which applies to rotation matrices as composition of rotations.

On the contrary, we normally consider the kinematically computed base velocity as:

$$
\boldsymbol{y}_{k}^{n}=\overbrace{{ }^{w} \boldsymbol{R}_{b}{ }^{b} \boldsymbol{v}_{b}+\boldsymbol{n}_{\boldsymbol{v}_{b}}}^{\boldsymbol{h}^{\boldsymbol{n}}\left(\boldsymbol{x}_{\boldsymbol{k}}\right)+\boldsymbol{n}_{\boldsymbol{v}_{\boldsymbol{b}}}}
$$

with $\boldsymbol{n}_{\boldsymbol{v}_{b}} \sim \mathcal{N}\left(\mathbf{0}, \boldsymbol{R}_{k}^{n}\right)$ be the normal zero mean kinematic velocity noise with covariance $\boldsymbol{R}_{k}^{n}$. The above measurements do not accumulate leg odometry drift during the gait and are commonly not contaminated with outliers when accurate contact states are estimated [30], [31]. Thus, we distinguish them with the superscript $n$ for nominal measurements that will be not examined for outliers.

To derive the linearization of Eq. (8) we consider the error exponential coordinates related with the external rotation [21], then:

$$
\boldsymbol{\delta} \boldsymbol{y}_{k}^{o}=\boldsymbol{H}_{k}^{o} \boldsymbol{\delta} \boldsymbol{x}_{k}+\boldsymbol{n}_{k}
$$

with

$$
\boldsymbol{H}_{k}^{o}=\left[\begin{array}{lllll}
\mathbf{0} & \mathbf{0} & \boldsymbol{I} & \mathbf{0} & \mathbf{0} \\
\mathbf{0} & \boldsymbol{I} & \mathbf{0} & \mathbf{0} & \mathbf{0}
\end{array}\right]
$$

On the other hand, the linearization of (9) is straightforward to compute:

$$
\boldsymbol{\delta} \boldsymbol{y}_{k}^{n}=\boldsymbol{H}_{k}^{n} \boldsymbol{\delta} \boldsymbol{x}_{k}+\boldsymbol{n}_{\boldsymbol{v}_{\boldsymbol{b}}}
$$

where

$$
\boldsymbol{H}_{k}^{n}=\left[\begin{array}{lllll}
{ }^{w} \boldsymbol{R}_{b} & -{ }^{w} \boldsymbol{R}_{b} \boldsymbol{v}_{b[x]} & \mathbf{0} & \mathbf{0} & \mathbf{0}
\end{array}\right]
$$

Subsequently, the ESKF update step is formulated as:

$$
\begin{aligned}
\boldsymbol{\delta} \boldsymbol{x}_{k} & =\boldsymbol{K}_{k}^{*}\left(\boldsymbol{y}_{k}^{*} \boxminus \boldsymbol{h}^{*}\left(\hat{\boldsymbol{x}}_{k}^{-}\right)\right) \\
\hat{\boldsymbol{x}}_{k}^{+} & =\hat{\boldsymbol{x}}_{k}^{-} \boxplus \boldsymbol{\delta} \boldsymbol{x}_{k} \\
\boldsymbol{P}_{k}^{+} & =\boldsymbol{P}_{k}^{-}-\boldsymbol{K}_{k}^{*}\left(\boldsymbol{H}_{k}^{*} \boldsymbol{P}_{k}^{-} \boldsymbol{H}_{k}^{* \top}+\boldsymbol{R}_{k}^{*}\right) \boldsymbol{K}_{k}^{* \top} \\
\boldsymbol{K}_{k}^{*} & =\boldsymbol{P}_{k}^{-} \boldsymbol{H}_{k}^{* \top}\left(\boldsymbol{H}_{k}^{*} \boldsymbol{P}_{k}^{-} \boldsymbol{H}_{k}^{* \top}+\boldsymbol{R}_{k}^{*}\right)^{-1}
\end{aligned}
$$

where the superscript * can be either $o$ or $n$ depending on the set of measurements considered.

\section{Outlier Detection}

In this section, the main result of this work is presented. The outlier detection framework presented in [27] is integrated with the ESKF to introduce a base estimator robust to outliers.

In order to detect outlier measurements, Wang et al. [27], utilized a beta-Bernouli distribution to probabilistically quantify whether a measurement is outlier or not. Beta-Bernouli distributions have been proved effective in various outlier resilient algorithms in the past [32], [33]. To this end, in [27] a binary indicator variable $z_{k}$ was introduced. Accordingly, when $z_{k}$ is one, $y_{k}^{o}$ is a nomimal measurement while when $z_{k}$ is zero, $y_{k}^{o}$ is an outlier. The latter can be formulated as:

$$
p\left(\boldsymbol{y}_{k}^{o} \mid \boldsymbol{x}_{k}, z_{k}\right)=\mathcal{N}\left(\boldsymbol{h}^{o}\left(\boldsymbol{x}_{k}\right), \boldsymbol{R}_{k}\right)^{z_{k}}
$$

Evidently, when $z_{k}=0$, Eq. (18) becomes a constant and cannot contribute to the state estimation, since the distribution is measurement independent.

Subsequently, in order to properly infer the indicator variable, a beta-Bernoulli hierarchical prior [34] is enforced. In such a way, $z_{k}$ is a Bernoulli variable influenced by $\pi_{k}$ :

$$
p\left(z_{k} \mid \pi_{k}\right)=\pi_{k}^{z_{k}}\left(1-\pi_{k}\right)^{\left(1-z_{k}\right)}
$$

where $\pi_{k}$ follows a beta distribution:

$$
p\left(\pi_{k}\right)=\frac{\pi_{k}^{e_{0}-1}\left(1-\pi_{k}\right)^{f_{0}-1}}{\mathcal{B}\left(e_{0}, f_{0}\right)}
$$

with $\mathcal{B}$ denoting the beta function, parametrized by $e_{0}$ and $f_{0}$.

Since, $z_{k}$ is modeled as a beta-Bernoulli variable, when the mean $\left\langle z_{k}\right\rangle$ is close to zero, e.g. $10^{-5}$, we treat the measurement as an outlier and ignore it, thus:

$$
\begin{aligned}
\hat{\boldsymbol{x}}_{k}^{+} & =\hat{\boldsymbol{x}}_{k}^{-} \\
\boldsymbol{P}_{k}^{+} & =\boldsymbol{P}_{k}^{-}
\end{aligned}
$$

otherwise, we weight the measurement noise $\boldsymbol{R}_{k}^{o}$ as:

$$
\boldsymbol{R}_{k}^{o}=\boldsymbol{R}_{k}^{o} /\left\langle z_{k}\right\rangle
$$

and perform the regular update as in (14)-(17).

The expectation of $z_{k}$ is computed in each iteration as follows:

$$
\left\langle z_{k}\right\rangle=\frac{p\left(z_{k}=1\right)}{p\left(z_{k}=1\right)+p\left(z_{k}=0\right)}
$$


with

$$
\begin{aligned}
& p\left(z_{k}=1\right)=c e^{\Psi\left(e_{k}\right)-\Psi\left(e_{k}+f_{k}\right)-\frac{1}{2} \operatorname{tr}\left(\boldsymbol{B}_{k} \boldsymbol{R}_{k}^{o-1}\right)} \\
& p\left(z_{k}=0\right)=c e^{\Psi\left(f_{k}\right)-\Psi\left(e_{k}+f_{k}\right)}
\end{aligned}
$$

where $c$ is the normalization constant to guarantee that (25), (26) are proper probabilities, $\Psi$ denotes the digamma function [34], and $\boldsymbol{B}_{k}$ is given by:

$$
\boldsymbol{B}_{k}=\int\left(\boldsymbol{y}_{k}^{o}-\boldsymbol{h}^{o}\left(\hat{\boldsymbol{x}}_{k}^{+}\right)\right)\left(\boldsymbol{y}_{k}^{o}-\boldsymbol{h}^{o}\left(\hat{\boldsymbol{x}}_{k}^{+}\right)\right)^{\top} p\left(\hat{\boldsymbol{x}}_{k}^{+}\right) d \boldsymbol{x}_{k}
$$

The integral in (27) is not straightforward to compute in the general nonlinear Gaussian case. In [27] the cubature rules [35] to obtain an approximate solution are used. In the context of the EKF, (27) can be derived analytically as:

$$
\begin{aligned}
\boldsymbol{B}_{k} & =\boldsymbol{y}_{k}^{o} \boldsymbol{y}_{k}^{o \top}-2 \boldsymbol{y}_{k}^{o} \boldsymbol{h}^{o}\left(\hat{\boldsymbol{x}}_{k}^{+}\right)^{\top} \\
& +\boldsymbol{h}^{o}\left(\hat{\boldsymbol{x}}_{k}^{+}\right) \boldsymbol{h}^{o}\left(\hat{\boldsymbol{x}}_{k}^{+}\right)^{\top}+\boldsymbol{H}_{k}^{o} \boldsymbol{P}_{k}^{+} \boldsymbol{H}_{k}^{o \top}
\end{aligned}
$$

The proof is given for completeness in the Appendix.

Finally, $e_{k}, f_{k}$ are updated in each iteration as:

$$
\begin{aligned}
e_{t} & =e_{0}+\left\langle z_{k}\right\rangle \\
f_{t} & =f_{0}+1-\left\langle z_{k}\right\rangle
\end{aligned}
$$

The proposed robust Gaussian ESKF (RGESKF) is summarized in Algorithm 1. We note, that no further knowledge of the measurement distribution [26] other than the covariance $\boldsymbol{R}_{k}^{o}$ is needed or empirically obtained thresholds as in [13] are required to perform outlier detection. The only tunable parameters are the beta-Bernoulli prior parameters $e_{0}$ and $f_{0}$. Experimentally, $e_{0}$ and $f_{0}$ have been set to 0.9 and 0.1 respectively. The latter values have been used in all conducted experiments, including real tests with the two robots (cf. Sec. III below) and in our open-source implementation [29]. As also stated in [27], we observed that the outlier detection process is insensitive to the latter parameters as long as $e_{0} /\left(e_{0}+f_{0}\right)$ is close to 1 . Presumably, this is the case, since it is more probable to observe a nomimal measurement rather than an outlier.

\section{RESULTS}

In this section, we outline representative results that demonstrate the accuracy and efficiency of the proposed scheme under real world conditions. Two actual humanoids were employed in our experiments, the full-size $29 \mathrm{DoF}$ WALK-MAN v2.0 humanoid [28] and a mini-size NAO robot. The WALK-MAN v2.0 robot uses the walking module introduced in [6], [36], with step time of $0.8 s$, using the XBotCore [37] and OpenSoT [38] control infrastructure in a $500 \mathrm{~Hz}$ control-loop. The walking module utilized with the NAO robot is based on [39] with a step time of $0.4 s$ and achieves a control-loop of $100 \mathrm{~Hz}$. The IMUs noise standard deviations used in our experiments are shown in Table I. For the WALK-MAN v2.0 VectorNav VN-100 IMU, we employed the noise densities given by the manufacturer at $200 \mathrm{~Hz}$, while for the IMU utilized in the NAO experiments an Allan variance analysis [40] was performed with 13 hour stationary data at $100 \mathrm{~Hz}$ to properly derive the values.

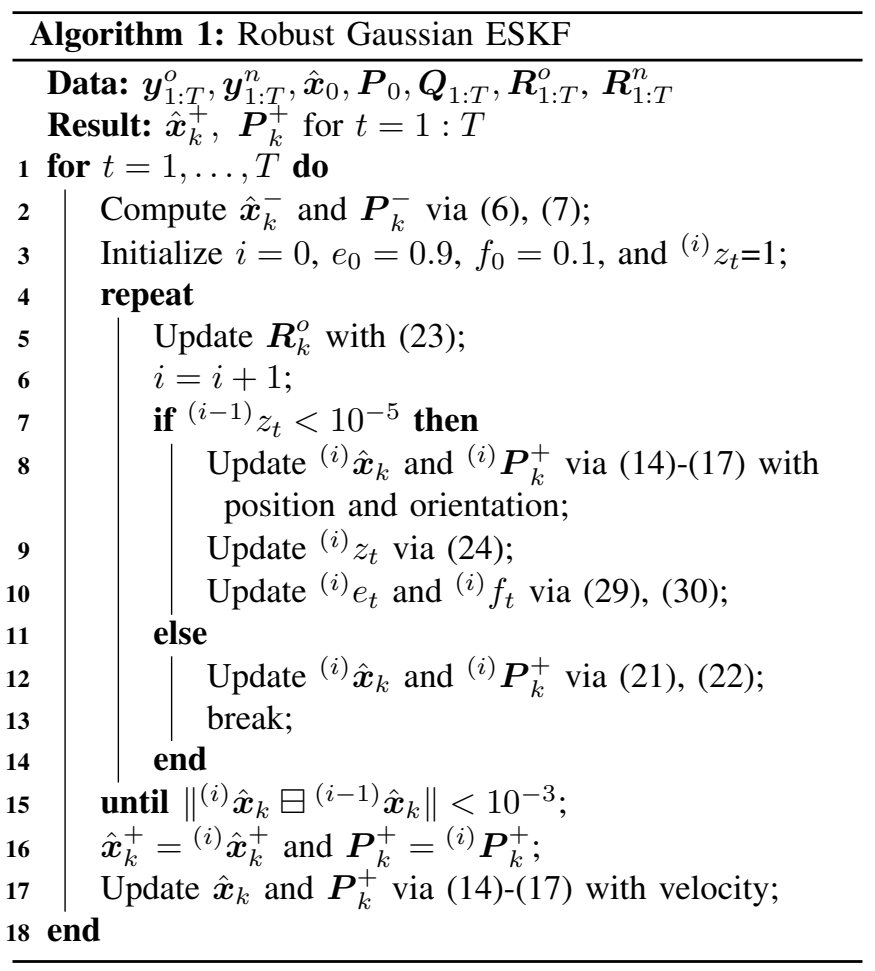

TABLE I

IMU NOISE STDS

\begin{tabular}{|c|c|c|c|c|}
\hline & $\boldsymbol{w}_{\boldsymbol{\omega}}\left(\frac{\mathrm{rad}}{\mathrm{s}}\right)$ & $\boldsymbol{w}_{\boldsymbol{\alpha}}\left(\frac{\mathrm{m}}{s^{2}}\right)$ & $\boldsymbol{w}_{\boldsymbol{b} \boldsymbol{\omega}}\left(\frac{\mathrm{rad}}{s^{2}}\right)$ & $\boldsymbol{w}_{\boldsymbol{b} \boldsymbol{\alpha}}\left(\frac{m}{s^{3}}\right)$ \\
\hline WALK-MAN & $9.77 \mathrm{e}-4$ & $2.21 \mathrm{e}-2$ & $1.53 \mathrm{e}-5$ & $2.43 \mathrm{e}-4$ \\
\hline NAO & $5.63 \mathrm{e}-3$ & $1.58 \mathrm{e}-2$ & $9.66 \mathrm{e}-4$ & $4.33 \mathrm{e}-3$ \\
\hline
\end{tabular}

Additionally, joint angle measurements are available at $200 \mathrm{~Hz}$ for the WALK-MAN v2.0 robot and at $100 \mathrm{~Hz}$ for NAO.

To facilitate a quantitative assessment, we compare the proposed RGESKF, to the ESKF without outlier detection, and to an ESKF where the outlier detection method in [14] is employed. The latter is termed as ESKF-TH since in each experiment we had to fine tune in advance a probabilistic threshold TH to achieve accurate detection according to the Mahalanobis distance $d_{M}$ :

$$
d_{M}=\left(\boldsymbol{y}_{k}^{o}-\boldsymbol{h}^{o}\left(\hat{\boldsymbol{x}}_{k}^{-}\right)\right)^{\top} \boldsymbol{S}_{k}^{-1}\left(\boldsymbol{y}_{k}^{o}-\boldsymbol{h}^{o}\left(\hat{\boldsymbol{x}}_{k}^{-}\right)\right)
$$

with

$$
\boldsymbol{S}_{k}=\boldsymbol{H}_{k}^{o} \boldsymbol{P}_{k}^{+} \boldsymbol{H}_{k}^{o \top}+\boldsymbol{R}_{k}^{o}
$$

In all our experiments, the aforementioned estimation schemes achieved real-time execution at the corresponding IMU rates.

\section{A. VO Outliers}

In the case of VO, we conducted two independent experiments, one with the WALK-MAN v2.0 while the groundtruth was recorded with an OptiTrack motion capture system and another one with a NAO robot navigating to a desired position in space. In the former case the PointGrey BlackFly 

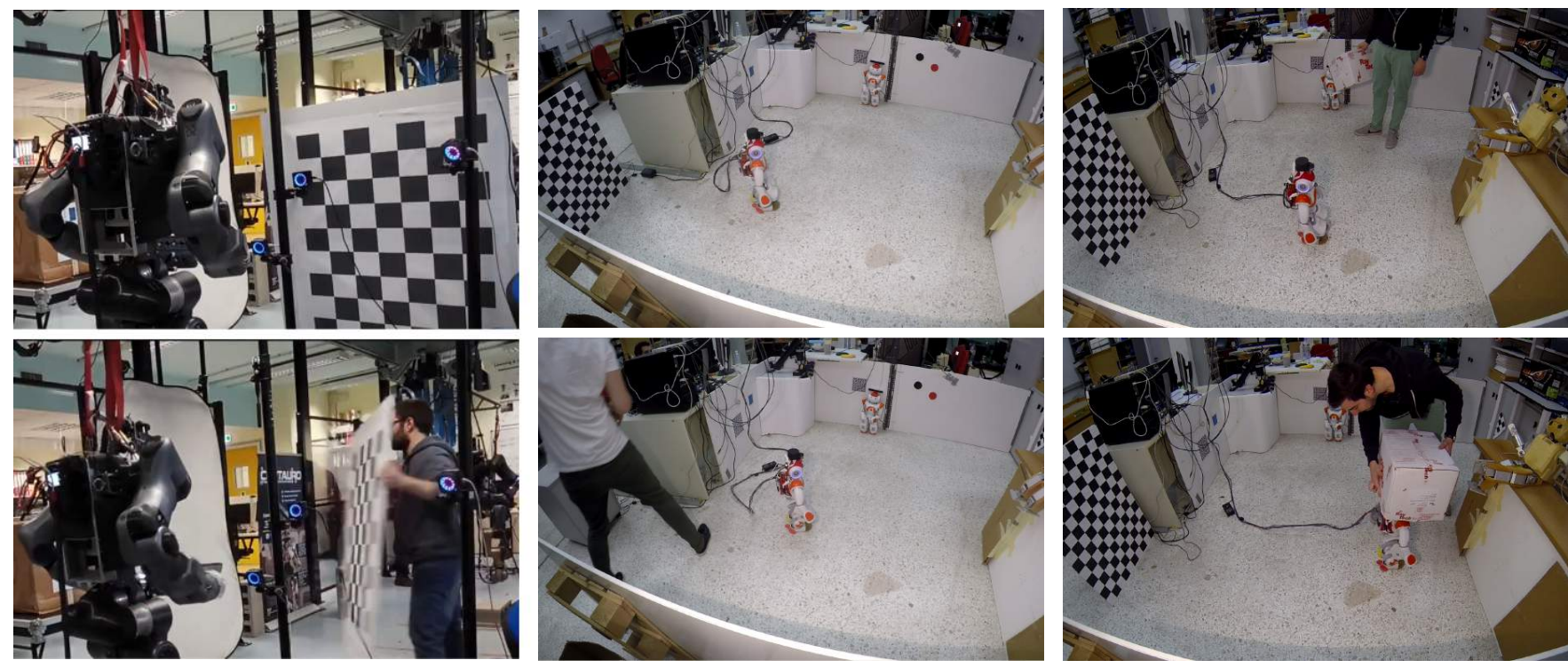

Fig. 2. Illustration of conducted experiments - Left: WALK-MAN v2.0 and VO outliers, Middle: NAO and VO outliers, Right: NAO and LO outliers.
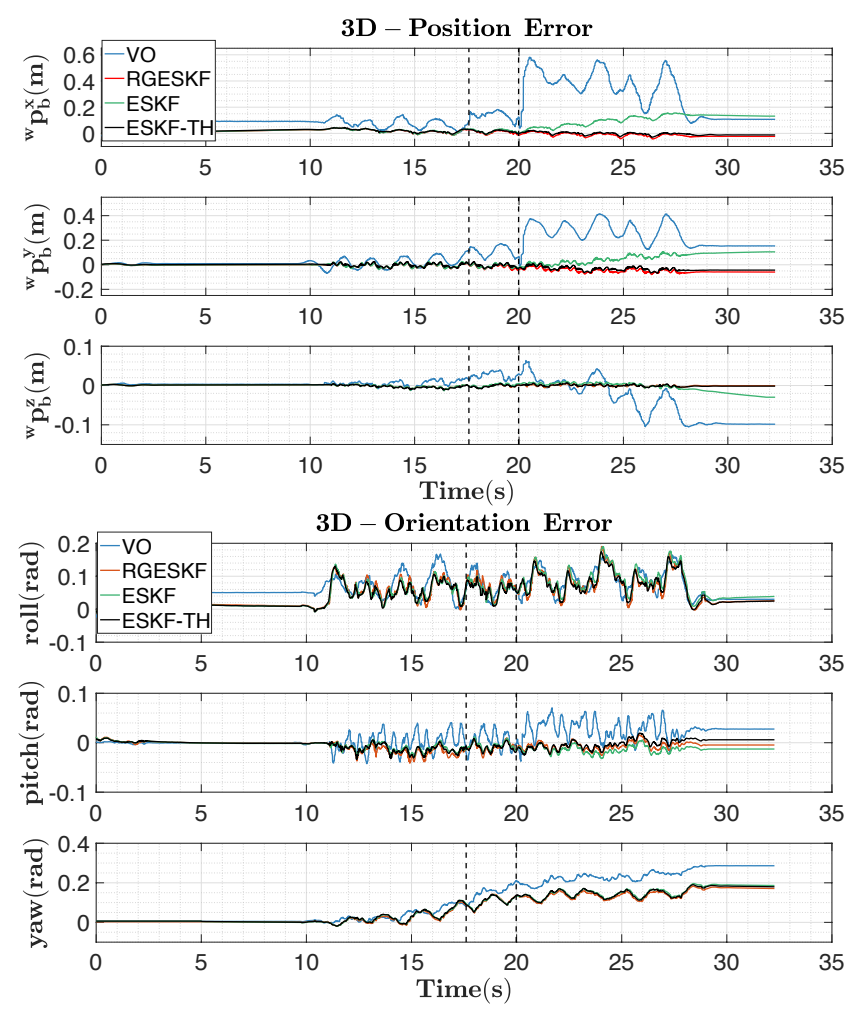

Fig. 3. Top: 3D - position error, Bottom: 3D - orientation error for the WALKMAN 2.0 - VO experiment, blue lines indicate the VO, red lines the proposed Robust Gaussian ESKF, green lines the ESKF, and black lines the finely tuned ESKF-TH. The first vertical dotted line corresponds to when a human enters the FoV of the camera, while the second one when he removes a strong feature source for $\mathrm{VO}$ which diverges.

BFLY-U3-23S6C camera at 40fps and 1080p resolution was used while in the latter, the Matrix-Vision mvBlueFOXMLC-200w running at 30fps and VGA was utilized. Both cameras are monocular and global-shutter. To obtain the VO

in both cases we used SVO [23]. The measurement noise standard deviations used in our VO experiments, are listed in Table II.

TABLE II

VO/LO And Kinematic Measurement Noise StDS

\begin{tabular}{|c|c|c|c|}
\hline & $\boldsymbol{n}_{\boldsymbol{p}_{b}}(\mathrm{~m})$ & $\boldsymbol{n}_{b}(\mathrm{rad})$ & $\boldsymbol{n}_{\boldsymbol{v}_{b}}\left(\frac{\mathrm{m}}{s}\right)$ \\
\hline WALK-MAN - VO & 0.035 & 0.05 & 0.015 \\
\hline NAO - VO & 0.04 & 0.05 & 0.013 \\
\hline NAO - LO & 0.04 & 0.05 & 0.013 \\
\hline
\end{tabular}

In the WALK-MAN v2.0 experiment, shown in Fig. 2 (left), a human unexpectedly crosses the Field of View (FoV) of the robot, causing sudden changes in the image intensity levels, and furthermore removes an object that is a strong feature source for VO. As a result, the scene changes rather drastically and henceforth, VO diverges while generating consecutive outlier measurements.

In Figure 3, the estimated 3D position and orientation errors w.r.t ground-truth for all three employed schemes is illustrated. Notice, at $t=17.6 \mathrm{~s}$ where the human appears in the FoV, $\mathrm{VO}$ starts to misbehave and at $t=19.9$ when static scene changes, VO eventually diverges. Consequently, the ESKF without outlier detection diverges as well and thus large positional errors are recorded. Nevertheless, this is not the case for the RGESKF and the ESKF-TH, where low errors were observed for both the position and the orientation. To finely tune the ESKF-TH, we had to run the filter and $\log$ the Mahalanobis distances (31), in order to determine a proper threshold $\mathrm{TH}$. A value of $\mathrm{TH}=23$ achieved the lowest estimation error. On the contrary our approach, RGESKF, which does not rely on finely tuned threshold or prior knowledge on the measurements, achieved very accurate and similar results to the tuned in advanced ESKF-TH. The Root-Mean Square Error (RMSE) for this experiment is shown in Table III. All employed schemes 
3D - Position Error
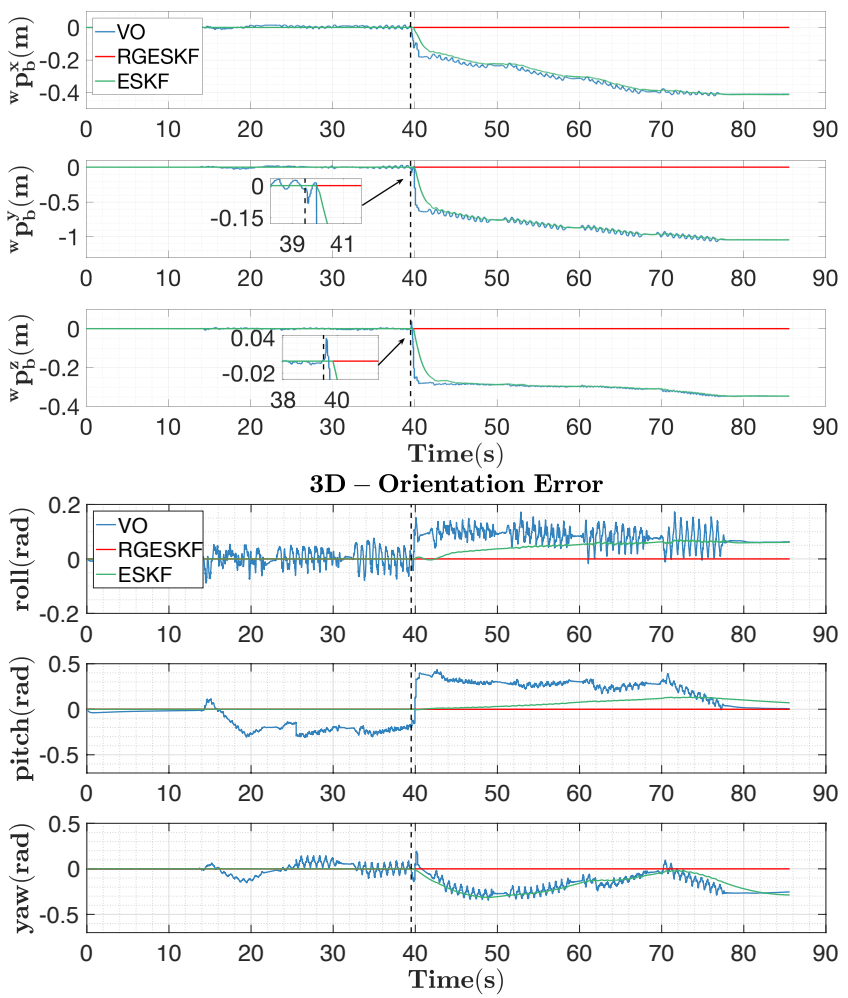

Fig. 4. Top: 3D - position error, Bottom: 3D - orientation error for the NAO - VO experiment, blue lines indicate the VO, red lines the proposed Robust Gaussian ESKF, and green lines the ESKF. The vertical dotted line specifies when a human removes a strong feature source for $\mathrm{VO}$ which diverges.

realized errors in the case of yaw estimation since it is unobservable [13], [15].

TABLE III

RMSE FOR THE WALK-MAN V2.0 VO EXPERIMENT

\begin{tabular}{|c|c|c|c|c|c|c|}
\hline & ${ }^{w} \boldsymbol{p}_{b}^{x}$ & ${ }^{w} \boldsymbol{p}_{b}^{y}$ & ${ }^{w} \boldsymbol{p}_{b}^{z}$ & roll & pitch & yaw \\
\hline VO & 0.217 & 0.162 & 0.043 & 0.075 & 0.022 & 0.160 \\
\hline ESKF & 0.071 & 0.045 & 0.009 & 0.066 & 0.011 & 0.105 \\
\hline RGESKF & 0.018 & 0.033 & 0.003 & 0.062 & 0.011 & 0.098 \\
\hline ESKF-TH & 0.017 & 0.025 & 0.003 & 0.059 & 0.010 & 0.102 \\
\hline
\end{tabular}

Similarly in the NAO's case, a human, present in the scene removes a strong feature source for $\mathrm{VO}$, while the robot is walking. For clarity, this is illustrated in Fig. 2 (middle). Since, we do not have ground-truth data available for the NAO experiments and given that a fine tuned ESKFTH can yield pretty accurate estimation, as evident by our previous experiment, we assume it as baseline to compare to. Subsequently, to properly derive the threshold needed, we computed (31) at $t=39.6 \mathrm{~s}$, the exact time when the human changes the scenery. The previous, was found to be $\mathrm{TH}=16$. Figure 4 demonstrates the $3 \mathrm{D}$ position and orientation error w.r.t the ESKF-TH. As illustrated, the ESKF realizes large errors for both positional and rotational quantities, when VO diverges at $t=39.6 \mathrm{~s}$. However, once again the RGESKF yielded practically identical results to the
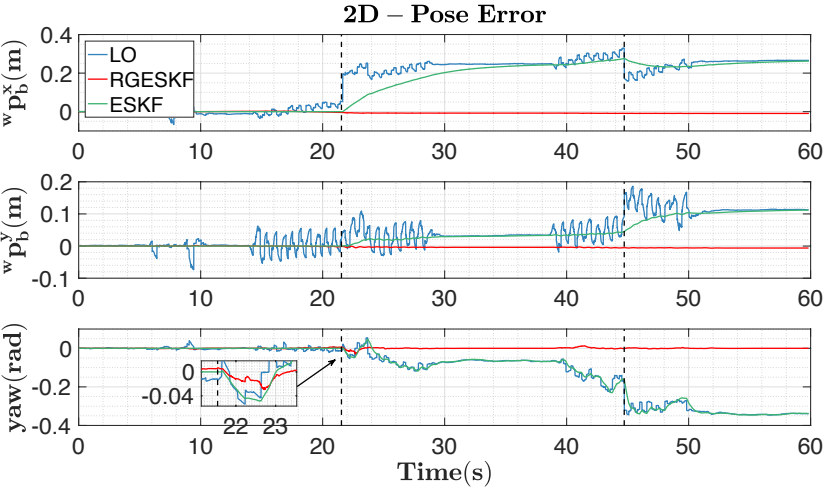

Fig. 5. 2D - pose error, for the NAO - LO experiment, blue lines indicate the LO, red lines the proposed Robust Gaussian ESKF, and green lines the ESKF. The vertical dotted lines corresponds to when a human suddenly covers the robot's LiDAR with a box, causing LO to diverge.

ESKF-TH as also evident in Table IV, where the RMSE is presented.

TABLE IV

RMSE W.R.T ESKF-TH FOR THE NAO VO EXPERIMENT

\begin{tabular}{|c|c|c|c|c|c|c|}
\hline & ${ }^{w} \boldsymbol{p}_{b}^{x}$ & ${ }^{w} \boldsymbol{p}_{b}^{y}$ & ${ }^{w} \boldsymbol{p}_{b}^{z}$ & roll & pitch & yaw \\
\hline VO & 0.246 & 0.653 & 0.227 & 0.066 & 0.246 & 0.152 \\
\hline ESKF & 0.238 & 0.643 & 0.223 & 0.037 & 0.061 & 0.146 \\
\hline RGESKF & $2.8 \mathrm{e}-6$ & $2.6 \mathrm{e}-7$ & $2.3 \mathrm{e}-6$ & $6.8 \mathrm{e}-7$ & $1.6 \mathrm{e}-6$ & $9.2 \mathrm{e}-6$ \\
\hline
\end{tabular}

\section{B. LO Outliers}

Next, we examine how LO outlier measurements can degrade the estimation performance. To do so, we utilize an RP-LiDAR360 mounted on NAO's head to obtain planar scans every $5 \mathrm{~Hz}$. Subsequently, we employed RF2O [24] to compute the $2 \mathrm{D}$ pose e.g. position $\mathrm{x}, \mathrm{y}$ and yaw, with scan matching. The measurement noise assumed in this experiment is shown at Table II.

In order to generate LO outliers, a human covers the spinning laser while NAO walks, as depicted in Fig. 2 (right). This corresponds to the scenario where a robot in motion is suddenly surrounded by people. To this end, the static world used to derive the LO is drastically changed which in turn gives rise to outliers.

In the conducted experiment, NAO is commanded to walk straight, stop, and then walk straight again. While walking a human covers the LiDAR twice to generate LO outliers. As previously, we compare the estimation results to the ESKF$\mathrm{TH}$, which we have accurately tuned in advance as before. A threshold of $\mathrm{TH}=9$ was experimentally found to be sufficient for this specific experiment. The $2 \mathrm{D}$ pose error is shown in Figure 5. Time $t=21.5 \mathrm{~s}$ marks the instant where the human covers the LiDAR for the first time. At that time, a large jump in the LO position in $\mathrm{x}$ axis is recorded which in turn causes large error to the ESKF estimation in the same axis. Subsequently, after $23.5 \mathrm{~s}$ the human covers the LiDAR one more time. This time larger errors are evident in the base's $y$ position and the base's yaw angle causing again the ESKF to misbehave. Interestingly, the proposed scheme was 
proven to be robust and automatically ignore the inaccurate LO measurements. The RMSE for this particular experiment is indicated in Table V. As demonstrated, the RGESKF yields a similar estimation result for all quantities of interest when compared to a finely tuned ESKF-TH.

TABLE V

RMSE W.R.T ESKF-TH FOR THE NAO LO EXPERIMENT

\begin{tabular}{|c|c|c|c|}
\hline & ${ }^{w} \boldsymbol{p}_{b}^{x}$ & ${ }^{w} \boldsymbol{p}_{b}^{y}$ & yaw \\
\hline LO & 0.197 & 0.066 & 0.174 \\
\hline ESKF & 0.180 & 0.054 & 0.173 \\
\hline RGESKF & 0.004 & 0.002 & 0.006 \\
\hline
\end{tabular}

All our experiments are illustrated in high quality at https://youtu.be/ojogeY3xSsw

\section{DISCUSSION}

As evident, the proposed RGESKF is characterized by high accuracy and strong outlier rejection capabilities. The latter hold true, even when consecutive VO/LO outlier measurements were observed. Additionally, no prior knowledge of the measurement distributions [26] or finely tuned thresholds [14] are required for the success of the proposed scheme. On the contrary, notice that the ESKF-TH needed three different thresholds, one for each experiment, to achieve accurate performance. This is evident by (31), where an optimal threshold depends on the measurement noise $\boldsymbol{R}_{k}^{o}$ and the error-state uncertainty $\boldsymbol{P}_{k}^{+}$. In addition, in all our $\mathrm{VO} / \mathrm{LO}$ experiments, the outlier detection part in Algorithm 1 took at most three iterations to complete. Thus, in the opensource released implementation [29], we loop three times instead of computing in every iteration the condition in line 15. Moreover, it is noteworthy that in the VO experiments the initial derived SVO orientation can be erroneous. We suspect this is probably due to a) inaccurate scale initialization, b) imperfect extrinsic and intrinsic calibrations. Nevertheless, as also seen in the results, this does not degrade the estimation accuracy since the fused IMU and kinematically computed base velocity measurements also carry information that contribute to the orientation estimation. Furthermore, it is important to clarify that the RGESKF does not only detect and reject outliers as the ESKF-TH does, but automatically weights the measurement noise according to (23) in order to avoid information loss when non-ideal/non-outlier measurements arrive. Finally, the proposed method can be appropriately employed to other robotic platforms, such as Unmanned Aerial Vehicles (UAVs), which also utilize the ESKF [41] for state estimation.

\section{CONCLuSion}

Prominent examples of VO/LO approaches readily assume that the world in which the robot acts, is static. Nevertheless, to enable humanoids co-exist with humans in dynamically changing environments, their state-estimation schemes must be robustified. In this work, we tackled the presence of VO/LO outlier measurements in base estimation by proposing the RGESKF. After mathematically establishing the proposed scheme, we provided a quantitative and qualitative assessment with two robots, namely a full-size WALK-MAN v2.0 humanoid and a mini-size NAO robot, demonstrating the accuracy and efficiency of the proposed scheme in real-world conditions. Finally, in order to reinforce further research endeavours, we release our implementation as an open-source ROS/C++ package [29].

Planned future work regards, utilizing the proposed scheme in humanoid navigation in human/gradually changing environments, where outliers are harder to detect [42]. In addition, we will investigate the estimation performance in locomotion-manipulation tasks, where the humanoid has to walk to a desired location and grasp an object. Commonly while grasping, multiple self-parts appear in the FoV and possibly give rise to outliers. Moreover, it would be interesting to compare the proposed method to other state estimation schemes that directly include leg contacts [15]. Finally, we will address how to recover from $\mathrm{VO} / \mathrm{LO}$ divergence in order to continue integrating $\mathrm{VO} / \mathrm{LO}$ measurements.

\section{APPENDIX}

$$
\begin{aligned}
\boldsymbol{B}_{k} & =\int\left(\boldsymbol{y}_{k}^{o}-\boldsymbol{h}^{o}\left(\boldsymbol{x}_{k}\right)\right)\left(\boldsymbol{y}_{k}^{o}-\boldsymbol{h}^{o}\left(\boldsymbol{x}_{k}\right)\right)^{\top} p\left(\hat{\boldsymbol{x}}_{k}\right) d \boldsymbol{x}_{k} \\
& =\boldsymbol{y}_{k}^{o} \boldsymbol{y}_{k}^{o \top}-2 \boldsymbol{y}_{k}^{o} \int \boldsymbol{h}^{o}\left(\boldsymbol{x}_{k}\right)^{\top} p\left(\hat{\boldsymbol{x}}_{k}\right) d \boldsymbol{x}_{k} \\
& +\int \boldsymbol{h}^{o}\left(\boldsymbol{x}_{k}\right) \boldsymbol{h}^{o}\left(\boldsymbol{x}_{k}\right)^{\top} p\left(\hat{\boldsymbol{x}}_{k}\right) d \boldsymbol{x}_{k}
\end{aligned}
$$

using the first order approximation of (8) post to the update:

$$
\boldsymbol{h}^{o}(\boldsymbol{x})=\boldsymbol{h}\left(\hat{\boldsymbol{x}}_{k}^{+}\right)+\boldsymbol{H}_{k}^{o}\left(\boldsymbol{x}_{t}-\hat{\boldsymbol{x}}_{k}^{+}\right)
$$

the first integral of (33) can be computed as:

$$
\begin{aligned}
& \int \boldsymbol{h}^{o}\left(\boldsymbol{x}_{k}\right)^{\top} p\left(\hat{\boldsymbol{x}}_{k}\right) d \boldsymbol{x}_{k}=\int \boldsymbol{h}^{o}\left(\hat{\boldsymbol{x}}_{k}^{+}\right)^{\top} p\left(\hat{\boldsymbol{x}}_{k}\right) d \boldsymbol{x}_{k} \\
& +\int\left(\boldsymbol{x}_{t}-\hat{\boldsymbol{x}}_{k}^{+}\right)^{\top} \boldsymbol{H}_{k}^{o \top} p\left(\hat{\boldsymbol{x}}_{k}\right) d \boldsymbol{x}_{k}=\boldsymbol{h}^{o}\left(\hat{\boldsymbol{x}}_{k}^{+}\right)^{\top}
\end{aligned}
$$

while the second integral of (33) is equivalent to:

$$
\begin{gathered}
\int \boldsymbol{h}^{o}\left(\boldsymbol{x}_{k}\right) \boldsymbol{h}^{o}\left(\boldsymbol{x}_{k}\right)^{\top} p\left(\hat{\boldsymbol{x}}_{k}\right) d \boldsymbol{x}_{k}=\int\left(\hat{\boldsymbol{x}}_{k}^{+}+\boldsymbol{H}_{k}^{o}\left(\boldsymbol{x}_{k}-\hat{\boldsymbol{x}}_{k}^{+}\right)\right) \\
\left(\hat{\boldsymbol{x}}_{k}^{+}+\boldsymbol{H}\left(\boldsymbol{x}_{t}-\hat{\boldsymbol{x}}_{k}^{+}\right)\right)^{\top} p\left(\hat{\boldsymbol{x}}_{k}\right) d \boldsymbol{x}_{k}=\hat{\boldsymbol{x}}_{k}^{+} \hat{\boldsymbol{x}}_{k}^{+\top}+\boldsymbol{H}_{k}^{o} \boldsymbol{P}_{k}^{+} \boldsymbol{H}_{k}^{o \top}
\end{gathered}
$$

Thus (33) becomes:

$$
\begin{aligned}
\boldsymbol{B}_{k} & =\boldsymbol{y}_{k}^{o} \boldsymbol{y}_{k}^{o \top}-2 \boldsymbol{y}_{k}^{o} \boldsymbol{h}^{o}\left(\hat{\boldsymbol{x}}_{k}^{+}\right)^{\top} \\
& +\boldsymbol{h}^{o}\left(\hat{\boldsymbol{x}}_{k}^{+}\right) \boldsymbol{h}^{o}\left(\hat{\boldsymbol{x}}_{k}^{+}\right)^{\top}+\boldsymbol{H}_{k}^{o} \boldsymbol{P}_{k}^{+} \boldsymbol{H}_{k}^{o \top}
\end{aligned}
$$

\section{ACKNOWLEDGMENT}

The authors would like to thank Hongwei Wang for his helpful discussion on the topic examined in this paper. 


\section{REFERENCES}

[1] B. Aceituno-Cabezas, C. Mastalli, H. Dai, M. Focchi, A. Radulescu, D. G. Caldwell, J. Cappelletto, J. C. Grieco, G. Fernndez-Lpez, and C. Semini, "Simultaneous contact, gait, and motion planning for robust multilegged locomotion via mixed-integer convex optimization," IEEE Robotics and Automation Letters, vol. 3, no. 3, pp. 2531-2538, July 2018.

[2] A. W. Winkler, C. D. Bellicoso, M. Hutter, and J. Buchli, "Gait and trajectory optimization for legged systems through phase-based endeffector parameterization," IEEE Robotics and Automation Letters, vol. 3, no. 3, pp. 1560-1567, July 2018.

[3] A. Hereid, C. M. Hubicki, E. A. Cousineau, and A. D. Ames, "Dynamic Humanoid Locomotion: A Scalable Formulation for HZD Gait Optimization," IEEE Transactions on Robotics, 2018.

[4] M. Neunert, M. Stuble, M. Giftthaler, C. D. Bellicoso, J. Carius, C. Gehring, M. Hutter, and J. Buchli, "Whole-body nonlinear model predictive control through contacts for quadrupeds," IEEE Robotics and Automation Letters, vol. 3, no. 3, pp. 1458-1465, July 2018.

[5] A. Herzog, N. Rotella, S. Mason, F. Grimminger, S. Schaal, and L. Righetti, "Momentum control with hierarchical inverse dynamics on a torque-controlled humanoid," Auton. Robots, vol. 40, no. 3, pp. 473-491, Mar. 2016.

[6] C. Zhou, X. Wang, Z. Li, and N. Tsagarakis, "Overview of Gait Synthesis for the Humanoid COMAN," vol. 14, no. 1, 2017, pp. 1525.

[7] T. Flayols, A. Del Prete, P. Wensing, A. Mifsud, M. Benallegue, and O. Stasse, "Experimental evaluation of simple estimators for humanoid robots," in 2017 IEEE-RAS 17th International Conference on Humanoid Robotics (Humanoids), Nov 2017, pp. 889-895.

[8] S. Kuindersma, R. Deits, M. Fallon, A. Valenzuela, H. Dai, F. Permenter, T. Koolen, P. Marion, and R. Tedrake, "Optimization-based Locomotion Planning, Estimation, and Control Design for the Atlas Humanoid Robot," Autonomous Robots, vol. 40, pp. 429-455, 2016.

[9] M. F. Fallon, M. Antone, N. Roy, and S. Teller, "Drift-free Humanoid State Estimation Fusing Kinematic, Inertial and LIDAR Sensing," in IEEE-RAS International Conference on Humanoid Robots, 2014, pp. 112-119.

[10] M. F. Fallon, P. Marion, R. Deits, T. Whelan, M. Antone, J. McDonald, and R. Tedrake, "Continuous Humanoid Locomotion over Uneven Terrain using Stereo Fusion," in IEEE-RAS Intl. Conf. on Humanoid Robots, 2015, pp. 881-888.

[11] V. Sushrutha Raghavan, D. Kanoulas, C. Zhou, D. G. Caldwell, and N. G. Tsagarakis, "A Study on Low-Drift State Estimation for Humanoid Locomotion, Using LiDAR and Kinematic-Inertial Data Fusion," in IEEE-RAS 18th International Conference on Humanoid Robots (Humanoids), 2018, pp. 1-8.

[12] M. Camurri, M. Fallon, S. Bazeille, A. Radulescu, V. Barasuol, D. G. Caldwell, and C. Semini, "Probabilistic contact estimation and impact detection for state estimation of quadruped robots," IEEE Robotics and Automation Letters, vol. 2, no. 2, pp. 1023-1030, April 2017.

[13] M. Bloesch, M. Hutter, M. Hoepflinger, S. Leutenegger, C. Gehring, C. D. Remy, and R. Siegwart, "State Estimation for Legged RobotsConsistent Fusion of Leg Kinematics and IMU ," Robotics Sci. and Sys., 2012.

[14] M. Bloesch, C. Gehring, P. Fankhauser, M. Hutter, M. A. Hoepflinger, and R. Siegwart, "State Estimation for Legged Robots on Unstable and Slippery Terrain," in IEEE/RSJ International Conference on Intelligent Robots and Systems, 2013, pp. 6058-6064.

[15] N. Rotella, M. Bloesch, L. Righetti, and S. Schaal, "State Estimation for a Humanoid Robot," in IEEE/RSJ Intl. Conf. on Intelligent Robots and Systems, 2014, pp. 952-958.

[16] N. Rotella, A. Herzog, S. Schaal, and L. Righetti, "Humanoid momentum estimation using sensed contact wrenches," in IEEE-RAS Intl. Conf. on Humanoid Robots, 2015, pp. 556-63.

[17] J. Carpintieri, M. Benallegue, N. Mansard, and J. P. Laumond, "Centerof-Mass Estimation for a Polyarticulated System in Contact - A Spectral Approach," IEEE Transactions on Robotics, vol. 32, 2016.

[18] S. Piperakis and P. Trahanias, "Non-linear ZMP based State Estimation for Humanoid Robot Locomotion," in IEEE-RAS Intl. Conf. on Humanoid Robots, 2016, pp. 202-209.

[19] Xinjilefu and C. G. Atkeson, "State Estimation of a Walking $\mathrm{Hu}-$ manoid Robot," in IEEE/RSJ Intl. Conf. on Intelligent Robots and Systems, 2012, pp. 3693-3699.
[20] R. Wittmann, A. Hildebrandt, D. Wahrmann, D. Rixen, and T. Buschmann, "State Estimation for Biped Robots using Multibody Dynamics," in IEEE/RSJ Conf. Intel. Robots and Systems, 2015.

[21] S. Piperakis, M. Koskinopoulou, and P. Trahanias, "Nonlinear State Estimation for Humanoid Robot Walking," IEEE Robotics and Automation Letters, vol. 3, no. 4, pp. 3347-3354, Oct 2018.

[22] J. Engel, V. Koltun, and D. Cremers, "Direct Sparse Odometry," IEEE Transactions on Pattern Analysis and Machine Intelligence, vol. 40, pp. 611-625, 2018.

[23] C. Forster, M. Pizzoli, and D. Scaramuzza, "SVO: Fast Semi-direct Monocular Visual Odometry," in IEEE Intl. Conf. on Robotics and Automation, 2014, pp. 15-22.

[24] M. Jaimez, J. G. Monroy, and J. Gonzalez-Jimenez, "Planar Odometry from a Radial Laser Scanner. A Range Flow-based Approach," in IEEE Intl. Conf. on Robotics and Automation, pp. 4479-4485.

[25] J. Zhang and S. Singh, "LOAM: Lidar Odometry and Mapping in Real-time," in Robotics: Science and Systems, 2014.

[26] R. Piche, S. Sarkka, and J. Hartikainen, "Recursive Outlier-robust Filtering and Smoothing for Nonlinear Systems using the Multivariate Student-t Distribution,' in IEEE International Workshop on Machine Learning for Signal Processing, 2012, pp. 1-6.

[27] H. Wang, H. Li, J. Fang, and H. Wang, "Robust Gaussian Kalman Filter With Outlier Detection," IEEE Signal Processing Letters, vol. 25, no. 8, pp. 1236-1240, 2018.

[28] N. G. Tsagarakis et al., "WALK-MAN: A High-Performance Humanoid Platform for Realistic Environments," Journal of Field Robotics, vol. 34, no. 7, pp. 1225-1259, 2017.

[29] S. Piperakis, "SEROW Humanoid Walking Estimator." [Online]. Available: https://github.com/mrsp/serow

[30] S. Piperakis, S. Timotheatos, and P. Trahanias, "Unsupervised Gait Phase Estimation for Humanoid Robot Walking," in IEEE Intl. Conf. on Robotics and Automation, 2019.

[31] N. Rotella, S. Schaal, and L. Righetti, "Unsupervised Contact Learning for Humanoid Estimation and Control," in Proceedings of the IEEE Intl. Conf. on Robotics and Automation, 2018.

[32] X. Ding, L. He, and L. Carin, "Bayesian Robust Principal Component Analysis," in IEEE Trans. Image Process, vol. 20, no. 12, 2011, pp. 3419-3430.

[33] Q. Wan, H. Duan, J. Fang, H. Li, and Z. Xing, "Robust Bayesian Compressed Sensing with Outliers," in Signal Processing, vol. 140, 2017, pp. 104-109.

[34] J. Paisley and L. Carin, "Nonparametric Factor Analysis with Beta Process Priors," in 26th Annual International Conference on Machine Learning. New York, NY, USA: ACM, 2009, pp. 777-784.

[35] I. Arasaratnam and S. Haykin, "Cubature Kalman Filters," IEEE Transactions on Automatic Control, vol. 54, no. 6, pp. 1254-1269, 2009.

[36] D. Kanoulas, C. Zhou, A. Nguyen, G. Kanoulas, D. G. Caldwell, and N. G. Tsagarakis, "Vision-based Foothold Contact Reasoning using Curved Surface Patches," in IEEE-RAS 17th International Conference on Humanoid Robotics (Humanoids), 2017, pp. 121-128.

[37] L. Muratore, A. Laurenzi, E. M. Hoffman, A. Rocchi, D. G. Caldwell, and N. G. Tsagarakis, "Xbotcore: A Real-Time Cross-Robot Software Platform," in IEEE International Conference on Robotic Computing (IRC), 2017, pp. 77-80.

[38] A. Rocchi, E. M. Hoffman, D. G. Caldwell, and N. G. Tsagarakis, "OpenSoT: A Whole-body Control Library for the Compliant $\mathrm{Hu}$ manoid Robot COMAN," in IEEE International Conference on Robotics and Automation (ICRA), 2015, pp. 6248-6253.

[39] S. Piperakis, E. Orfanoudakis, and M. Lagoudakis, "Predictive Control for Dynamic Locomotion of Real Humanoid Robots," in IEEE/RSJ Intl. Conf. Intel. Robots and Systems, 2014, pp. 4036-4043.

[40] N. El-Sheimy, H. Hou, and X. Niu, "Analysis and Modeling of Inertial Sensors Using Allan Variance," IEEE Transactions on Instrumentation and Measurement, pp. 140-149, 2008.

[41] A. Bry, A. Bachrach, and N. Roy, "State estimation for aggressive flight in gps-denied environments using onboard sensing," in IEEE International Conference on Robotics and Automation, 2012, pp. 1-8.

[42] S. Piperakis, N. Tavoularis, E. Hourdakis, D. Kanoulas, and P. Trahanias, "Humanoid Robot Dense RGB-D SLAM for Embedded Devices," in IEEE Intl. Conf. on Robotics and Automation (ICRA): 3rd Full-Day Workshop "Towards Real-World Deployment of Legged Robots“, 2019. 